\title{
Comparison of the Antibacterial Efficacy of Tea Tree Oil with $3 \%$ Sodium Hypochlorite and $2 \%$ Chlorhexidine against $E$. faecalis: An in vitro Study
}

Uday Kamath, Hina Sheth, Sai Ramesh, Keshav Singla

\begin{abstract}
Background and objectives: Among the bacterial species which most often cause endodontic failure, Enterococcus faecalis is the most important. This study compared the antibacterial efficacy of tea tree oil with $3 \%$ sodium hypochlorite and $2 \%$ chlorhexidine as a root canal irrigant, against $E$. faecalis.

Materials and methods: Normal saline, tea tree oil, $3 \%$ sodium hypochlorite, $2 \%$ chlorhexidine and fresh stains of $E$. faecalis were used. Four round wells, $4 \mathrm{~mm}$ deep and $8 \mathrm{~mm}$ diameter, were punched using sterile cork borer in blood agar plates and divided into four groups: group A: normal saline (control), group B: $3 \%$ sodium hypochlorite, group C: $2 \%$ chlorhexidine and group D: tea tree oil. Agar well diffusion method was performed to compare the antibacterial efficacy of tea tree oil, $3 \%$ sodium hypochlorite and $2 \%$ chlorhexidine.
\end{abstract}

Results: The results were tabulated and statistically analyzed using analysis of variance (ANOVA). Tea tree oil showed comparable inhibition of bacterial growth with sodium hypochlorite and chlorhexidine. No zone of inhibition was shown by normal saline (control).

Interpretation and conclusion: Tea tree oil showed statistically significant antimicrobial activity against Enterococcus faecalis, which is very much similar to sodium hypochlorite and chlorhexidine.

Abbreviations: CHX: Chlorhexidine; E. faecalis: Enterococcus faecalis; $\mathrm{NaOCl}$ : Sodium hypochlorite.

Keywords: Chlorhexidine, Enterococcus faecalis, Inhibition zone, Sodium hypochlorite, Tea tree oil.

How to cite this article: Kamath $U$, Sheth $H$, Ramesh $S$, Singla K. Comparison of the Antibacterial Efficacy of Tea Tree Oil with 3\% Sodium Hypochlorite and $2 \%$ Chlorhexidine against $E$. faecalis: An in vitro Study. J Contemp Dent 2013; 3(3):117-120.

\section{Source of support: Nil}

Conflict of interest: None declared

\section{INTRODUCTION}

The major objective in root canal treatment is to disinfect the entire root canal system. Although cleaning, shaping and use of antimicrobial medicaments are effective in reducing the bacterial load, some bacteria do remain behind and multiply, causing reinfection of the canal.

Enterococcus faecalis is the most commonly isolated species from the canals of teeth presenting post-treatment diseases. Enterococcus faecalis account for up to $77 \%$ of therapeutic failures. ${ }^{1}$ Residual pulpal tissue, bacteria and dentine debris may persist in the irregularities of root canal systems, even after meticulous mechanical preparation. ${ }^{2}$
Therefore, irrigant solutions should be used in combination with canal preparation. ${ }^{3}$

Root canal irrigants are used during chemomechanical procedures not only as antimicrobial agents but also to flush out loose debris, to lubricate the dentinal walls and to dissolve organic compounds in the canal. Sodium hypochlorite $(\mathrm{NaOCl})$ has been the most widely used root canal irrigating solution. The main advantages of sodium hypochlorite are its ability to dissolve necrotic tissue and its antibacterial properties against microorganisms. However, it has several undesirable characteristics, such as tissue toxicity, risk of emphysema, allergic potential and disagreeable smell and taste. ${ }^{4}$

The constant increase in antibiotic resistant strains and side effects caused by synthetic drugs has prompted researchers to look for herbal alternatives. Morinda citrifolia juice, ${ }^{5}$ neem leaf extract (Azadirachta indica), ${ }^{6}$ ginger, ${ }^{7}$ triphala and green tea polyphenols ${ }^{8}$ had been evaluated previously as endodontic irrigants.

Tea tree or Melaleuca alternifolia (Fig. 1) 4 is a native Australian plant, the oil of which has many properties that favor its use in dentistry. It is an antiseptic as well as an antifungal agent. It has mild solvent action and hence could hold potential applications in root canal treatment for dissolving the necrotic pulp tissue. Tea tree oil's major active component is terpinen-4-ol (typically 30-40\%). This compound is responsible for its antibacterial and antifungal properties. ${ }^{4}$

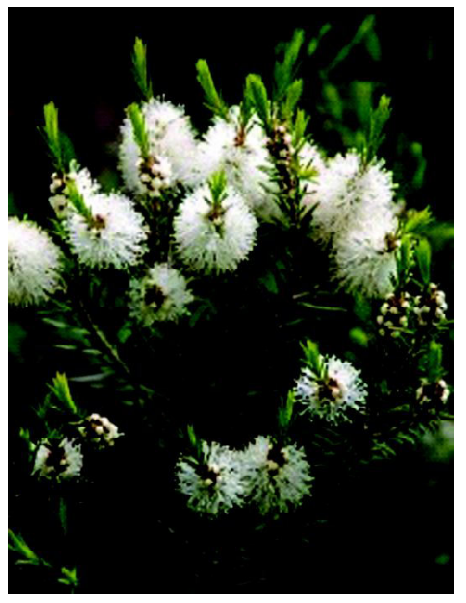

Fig. 1: Tea tree or Melaleuca alternifolia-native Australian plant (Source: Int J Drug Dev and Res 2011;3:68-77) 
This literature had shown that tea tree oil is having antimicrobial and therapeutic effects suggesting its potential to be used as an endodontic irrigant, but there is lack of much documentation or data regarding tea tree oil research in endodontics.

\section{AIMS AND OBJECTIVES}

The purpose of this in vitro study was to compare the antimicrobial activity of tea tree oil with $3 \%$ sodium hypochlorite and 2\% chlorhexidine ( $\mathrm{CHX}$ ) against E. faecalis, to assess the antimicrobial property of tea tree oil against endodontic pathogens, using the agar diffusion method.

\section{MATERIALS AND METHODS}

Tea tree oil was taken from Falcon Lab, Bengaluru (India), which was prepared by steam distillation of the foliage of Melaleuca alternifolia, then prepared to have miscibility in $85 \%(\mathrm{v} / \mathrm{v})$ ethanol at $20^{\circ} \mathrm{C}$, to get a concentration of $2 \%$ by volume. A total of $3 \%$ sodium hypochlorite (Venson India, Bengaluru) and 2\% chlorhexidine (Azure Laboratories Pvt Ltd, Kochi, India) were taken. Normal saline was used as control. The bacterial stock culture of Enterococcus faecalis (ATCC 29212) was obtained from Curewell Diagnostic Center, Ludhiana, India.

Culture was grown overnight in nutrient broth, adjusted to 0.5 turbidity reading on McFarland scale $(1.5 \times 108$ bacteria/ml $)$. Blood agar plates were taken and 'Lawn cultures' were prepared on the agar plates by swabbing the sterile swab across the media. Four round wells, $4 \mathrm{~mm}$ deep and $8 \mathrm{~mm}$ diameter, were punched using sterile cork borer, named as groups A, B, C, D. $50 \mu$ of the medicaments were added to the wells with the help of micropipette as follows:

- Group A: Normal saline (control)

- Group B: 3\% sodium hypochlorite

- Group C: $2 \%$ chlorhexidine

- Group D: Tea tree oil

A total of three such inoculation agar plates with medicament were prepared. Agar plates were incubated at $37^{\circ} \mathrm{C}$ for 24 hours in an incubator. The diameter of bacterial inhibition zones around each well was recorded to the nearest size in $\mathrm{mm}$ with the help of vernier caliper.

\section{RESULTS}

The results were tabulated and statistically analyzed using analysis of variance (ANOVA). Mean zone of inhibitions were recorded (Table 1). All three medicaments used in this study showed well-defined zones of inhibition around their respective wells, but no zone of inhibition was shown by normal saline (control) as shown in (Fig. 2). ANOVA showed that there was no significant difference between the zone of inhibition diameters of tea tree oil, $3 \%$ sodium hypochlorite and $2 \%$ chlorhexidine against E. faecalis. Maximum antimicrobial activity was shown by $2 \%$ chlorhexidine $(22.67 \mathrm{~mm})$, followed by tea tree oil $(22.33 \mathrm{~mm})$ and $3 \%$ sodium hypochlorite $(21.0 \mathrm{~mm})$ (Graph 1). Tea tree oil showed comparable zone of inhibitions to sodium hypochlorite and chlorhexidine.

\section{DISCUSSION}

Owing to the potential side effects, safety concerns and ineffectiveness of conventional allopathic formulations, consumption of natural remedies like neem, tulsi and tea tree oil, have increased over the last few decades. This approach may be termed as phytotherapeutics or ethnopharmacology. ${ }^{4}$

Sodium hypochlorite and chlorhexidine have varying degree of antibacterial properties. But, they have several undesirable characteristics, such as tissue toxicity, allergic potential and inability to remove smear layer.

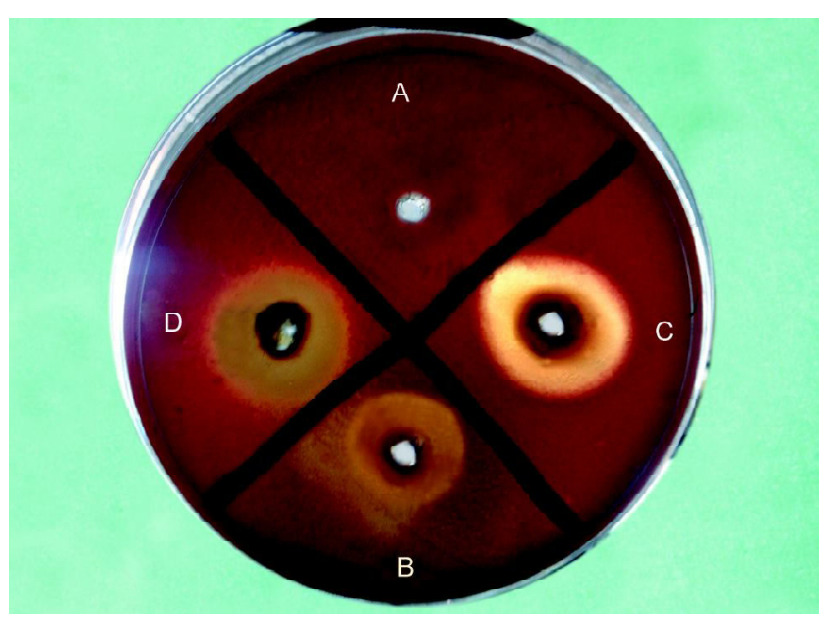

Fig. 2: Zone of inhibition: group A (normal saline)-0 mm, group B (sodium hypochlorite) $-20.06 \mathrm{~mm}$, group C (chlorhexidine) $-21.5 \mathrm{~mm}$, group D (tea tree oil)-22.46 mm

\begin{tabular}{clccccc}
\multicolumn{7}{c}{ Table 1: Readings for mean zones of inhibition in all groups } \\
Groups & Agent & $N$ & Mean & Range & Mean $\pm S D$ & ANOVA \\
\hline A & N. saline (control) & 3 & 0 & 0 & 0.00 & p $<0.001$ (Highly significant) \\
B & Sodium hypochlorite & 3 & 21.0 & $18-25$ & $21.00 \pm 3.61$ & \\
C & CHX & 3 & 22.67 & $21-25$ & $22.67 \pm 2.08$ & \\
D & Tea tree oil & 3 & 22.33 & $19-25$ & $22.33 \pm 3.06$ & \\
\hline
\end{tabular}

$\mathrm{N}$ : number of plates; SD: standard deviation; mean in $\mathrm{mm}$ 
Tea tree oil can be extract from the leaves and branches of Melaleuca alternifolia through distillation. It has antiseptic activities like, antibacterial, antifungal, antiviral, antitumor and anti-inflammatory. Oil of Melaleuca has a wide spectrum of activity that is mainly attributed to terpeno4-ol. It possesses effective antimicrobial activity against Gram-positive bacteria, such as Staphylococcus aureus and Gram-negative bacteria, such as Escherichia coli or Salmonella typhimurium. ${ }^{9}$

Mechanism of action is due to its hydrocarbon structure and attendant lipophilicity. Hydrocarbons do partition preferentially into biological membranes and disrupt their vital functions. This premise is further supported by data showing that tea tree oil causes lysis and the loss of membrane integrity and function by leakage of ions and the inhibition of respiration in microbes. ${ }^{10}$

The tea tree oil is believed to make an excellent wash for sore and weak eyes and also for the treatment of open sores and wounds due to its antiseptic, antifungal and mild solvent properties. ${ }^{11}$ In an in vitro study, it was shown that tea tree oil which might disinfect the root canal system as effective as sodium hypochlorite and toxicity of tea tree oil is lesser than sodium hypochlorite. ${ }^{12}$ Due to its properties, tea tree oil may have a role in the treatment of various infections. ${ }^{13}$ The recommended therapeutic concentration for tea tree oil is 2.5 to $5 \%$, in which it can retain its antibacterial property without any toxic effect. ${ }^{14}$

The major advantages of using herbal alternatives are easy availability, cost-effectiveness, increased shelf life, low toxicity, lack of microbial resistance.

This study showed that tea tree oil have good inhibitory efficacy against Enterococcus faecalis. It is very important to treat extrapolations of in vitro to in vivo studies with care. Other properties beyond antimicrobial activity must also be investigated before the final choice of an irrigant solution for clinical use, such as minimum inhibitory concentration,

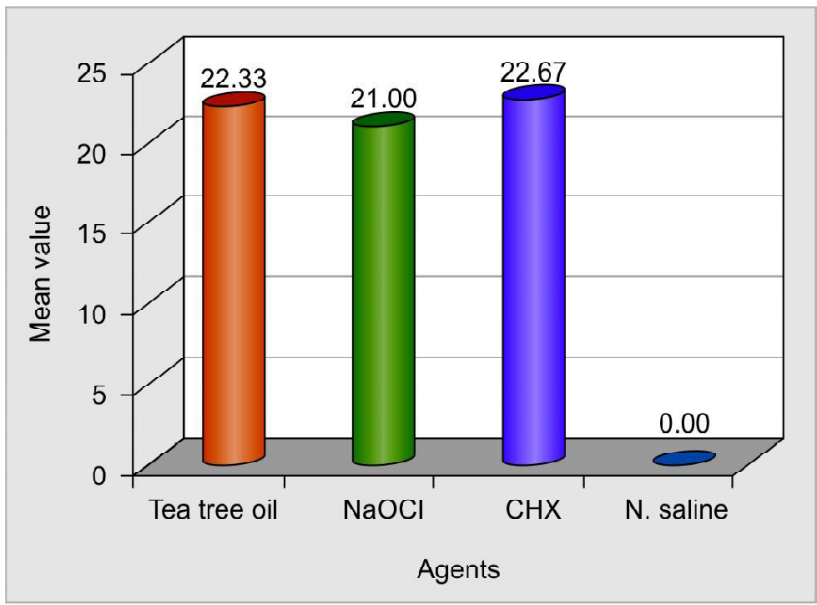

Graph 1: Mean inhibition zone in all groups tissue dissolution capacity, detoxification of endotoxin and acceptable biologic compatibility.

\section{CONCLUSION}

Under the limitations of this study, it was concluded that tea tree oil has a significant antimicrobial effect against E. faecalis. Microbial inhibition potential of tea tree oil observed in this study opens perspectives for its use as a root canal irrigant. However, preclinical and clinical trials are needed to evaluate biocompatibility and safety before tea tree oil can conclusively be recommended as an intracanal irrigating solution, but in vitro observation of tea tree oil efficacy appears promising.

\section{REFERENCES}

1. Siqueira JF, Rocas IN. Exploiting molecular methods to explore endodontic infections: Part 2-redefining the endodontic microbiota. J Endod 2005;31:488-498.

2. Segura JJ, Jimenez-Rubio A, Guerrero JM, Calvo JR. Comparative effects of two endodontic irrigants, chlorhexidine digluconate and sodium hypochlorite on macrophage adhesion to plastic surface. J Endod 1999;25:243-246.

3. D'Arcangelo C, Di Nardo Di Maio F, Stracci N, Spoto G, Malagnino VA, Caputi S. Pulp-dissolving ability of several endodontic irrigants: a spectrophotometric evaluation. Int $\mathbf{J}$ Immunopathol Pharmacol 2007;20(2):381-386.

4. Neelakantan P, Jagannathan N, Nazar N. Ethnopharmacological approach in endodontic treatment: a focused review. Int J Drug Devand Res 2011;3:68-77.

5. Murray PE, Farber RM, Namerow KN, Kuttler S, Godoy G. Evaluation of Morinda citrifolia as an endodontic irrigant. J Endod 2008;34:66-70.

6. Bohera A, Hegde V, Kokate S. Comparison of the antimiorobial efficiency of neem leaf extract and $2 \%$ sodium hypochloride against E. faecalis, A. albicans and mixed culture: an in vitro study. Endodont 2010;22:8-12.

7. Gulve NM, Gulve ND. Comparison of antimicrobial efficacy of ginger extract and 2\% sodium hypochlorite against Enterococcous faecalis using agar diffusion method. JIDA 2010;4(10):347-349.

8. Prabhakar J, Senthilkumar M, Priya MS, Mahalakshmi K, Sehgal PK, Sukumaran VG. Evaluation of antimicrobial efficacy of herbal alternatives (triphala and green teapolyphenols), MTAD, 5\% sodium hypochlorite against Enterococcus faecalis biofilm formed on tooth substrate: an in vitro study. J Endod 2010;36:83-86.

9. Ternes CE, Mello LL, Cruz AB. The influence of the essential oil of Melaleuca alternifolia on the healing of infected dental alveoli: a histological study in rats. Brazilian J Pharmacognosy 2007;17(3):349-355.

10. Carson CF, Hammer KA, Riley TV. Compilation and Review of Published and Unpublished Tea Tree Oil Literature: RIRDC Publication No. 05/151. Project No UWA-75A. Clin Microbiol Rev 2006;19(1):50-62.

11. Sadr Lahijani MS, RaoofKateb HR, Heady R, et al. The effect of German chamomile (Matricaria recutita L) extract and tea tree (Melaleuca alternifolia L) oil used as irrigants on removal of smear layer: a scanning electron microscopy study. Int Endod J 2006;39:190-195.

12. Parle M, Bansal N. Herbal medicines: Are they safe? Natural Product Radiance 2006;5:6-14. 
13. Carson CF, Mee BJ, Riley TV. Mechanism of action of Melaleuca alternifolia (tea tree) oil on Staphylococcus aureus determined by time-kill, lysis, leakage, and salt tolerance assays and electron microscopy. Antimicrobial Agents and Chemotherapy 2002;46,1914-1920.

14. Sharaf N, Aboul-Enein N, Hassan M. Effect of MTAD and tea tree oil irrigation before pulp capping on healing of exposed dental pulp. Australian J Basic and Applied Sciences 2012;6(7): 553-557.

\section{ABOUT THE AUTHORS}

\section{Uday Kamath (Corresponding Author)}

Head, Department of Conservative and Endodontics, Dr Syamala Reddy Dental College and Hospital, Bengaluru, Karnataka, India Phone: 9844027746, e-mail: m.uday.kamath@gmail.com

\section{Hina Sheth}

Professor, Department of Conservative and Endodontics Dr Syamala Reddy Dental College and Hospital, Bengaluru Karnataka, India

\section{Sai Ramesh}

Senior Lecturer, Department of Conservative and Endodontics Dr Syamala Reddy Dental College and Hospital, Bengaluru Karnataka, India

\section{Keshav Singla}

Postgraduate Student, Department of Conservative and Endodontics Dr Syamala Reddy Dental College and Hospital, Bengaluru Karnataka, India 